\title{
Extraction of Water Body Based on LandSat TM5 Imagery - A Case Study in the Yangtze River
}

\author{
Zhongshi Tang, Wenhao Ou, Yue Dai, and Yu Xin* \\ 3S Center, Tsinghua University, Beijing 100084, China \\ gisatsinghua. edu.cn
}

\begin{abstract}
A improved method named vegetation normalized difference water index (VNDWI) has been proposed in this paper based on NEW of Xiao Yun-fang(2010), which uses Band 1 and Band 7 and the normalized difference vegetation index (NDVI) in TM5 imagery to construct the VNDWI. This method has been tested in the Yangtze River Basin, and compares the results with other methods is the best. Due to the reflection characteristics differences between water and cloud, NEW method cannot distinguish the differences effectively. However, joins the NDVI parameter, VNDWI can enhance the difference between water and cloud, so it can remove clouds and its shadow noise from water body information without using complicated procedures, which is particular difficult work should be done in pretreatment.
\end{abstract}

Keywords: Extraction of Water Body, Imagery Processing, Binarization.

\section{Introduction}

The water body research is inseparable from the water scope and accurate extraction from the boundaries, it is particularly important to the coastline change monitoring, flood disaster monitoring, and disaster evaluation. In macro level, remote sensing monitoring of large scope area is having incomparable advantage. This paper extraction research based on the TM5 image of Yangtze River basin, and put forward the improved water extraction method.

Based on the research of water extraction from TM5 imagery, domestic and foreign scholars have made a lot of research, puts forward a variety of algorithm. In the foreign scholars' researches, 1990 Moller Jensen used the TM4 and TM5 imageries and set threshold to extract water[1], 1996 Mc Feeter using normalized water index NDWI = (TM2-TM4) / (TM2 + TM4) by inhibiting vegetation and soil information to extract water[2]. And domestic scholars Xu HanQiu proposed improved normalization water index MNDWI = (TM2-TM5) / $($ TM2 + TM5) in 2005, and set certain threshold to extract water [3]. In 2004 Chen HuaFang, put forward the method: (TM2 + TM3)-(T4-TM5) > k, which can be well separated by mountain shadow and water [4]. Xiao YanFang using the Band 1 and Band 7 in TM5 imagery for water information

* Corresponding author.

D. Li and Y. Chen (Eds.): CCTA 2012, Part II, IFIP AICT 393, pp. 416-420, 2013.

(C) IFIP International Federation for Information Processing 2013 
extraction [5], this method not only can extract water body like NDWI and MNDWI, but also has advantage in urban areas than other methods.

The above method has been widely used in practical work, but there are also have some disadvantages, such as the extraction of water information may contain cloud, vegetation and residents influences which may cause the inaccurate results, Salt and Pepper phenomenon as well.

\section{Materials and Methods}

In this paper, experimental data is TM5 imagery of the Yangtze River basin on July 5, 1988 from http://glcf.umiacs.umd.edu website. This image contains a complete water body, probably suit for verifying the precision of extraction of the water body by improved method.

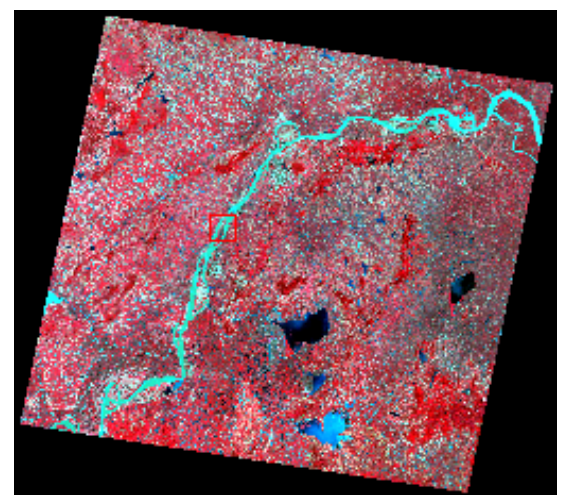

Fig. 1. Yangtze River Basin

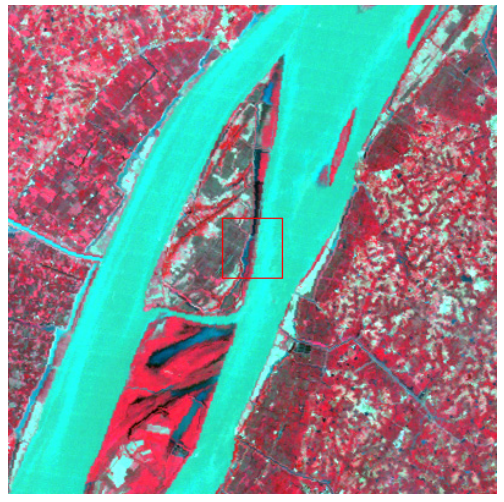

Fig. 2. Part of Yangtze River

Within the most scope of the spectrum from remote sensing sensors, water has a low reflection. For clear water, in the blue and green band reflectance reaches $4 \% \sim 5 \%$, from the red part of the reflectivity reaches $2 \% \sim 3 \%$, and in the near infrared and short wave infrared, almost all of the energy absorbed, because the characteristics of vegetation and soil formation is obviously, so often use red light band to recognize water body[6]. A normalized difference index of water named NDWI puts forward by Mc feeters, Chinese scholars Xu HanQiu put forward the modified normalization water index (MNDWI) and XiaoYanFang's NEW method is based on the principle from the differences in energy absorbing by different bands of sensor. However, Xiao YanFang's research indicates that band 1 and band 7 of TM5 imagery makes the minimum difference between water and buildings, and their sum is far higher than water, so it can be used to eliminate buildings and roads influence to the water body extraction.

But in actual study operation, the study area in the Yangtze River basin for this paper mentioned, buildings information may less than others, but cloud and its shadow are the main factors which may affect the accuracy of water extraction. For the cloud reflection characteristics of the bands in TM imageries, a statistic result table can be listed: 
Table 1. The Average Value of Water and Cloud

\begin{tabular}{lcccccccccc}
\hline & Band & Band2 & Band3 & Band4 & Band5 & Band7 & NDVI & NDWI & MNDWI & NEW \\
\hline Water & 106.5 & 56.9 & 35.9 & 25.1 & 14.6 & 9.6 & -0.391 & 0.346 & 0.986 & 0.997 \\
Cloud & 235.9 & 124.5 & 142.1 & 105.9 & 162.6 & 89.8 & -0.0366 & 0.0429 & 0.753 & 0.909 \\
\hline
\end{tabular}

From the table 1 we can see the largest difference of water and cloud is the NDVI value, so the proper way to distinguish between water and cloud is from NDVI value. This paper improves the NEW algorithm, join NDVI parameters, which named VNDWI here, the formula is:

$$
\text { VNDWI }=(\text { TM1-TM7) } /(\text { TM1 }+ \text { TM7) }>\mathrm{k} * \text { NDVI }
$$

(Formula 1)

The calculate results from formula 1 eliminates the influence of cloud and make the water body extraction more precisely, and buildings and subsequent shadow elimination is well preserved. Statistical analysis showed that the new method in this paper can extract the water body like NDWI, MNDWI and NEW, it also indicates more accurate extraction in the Yangtze River basin water information from filter out the part of the clouds and the clouds' shadow influences.

\section{Results and Discussion}

From the imagery of Yangtze River basin, we can see some clouds and the shadow over the water body. In order to show water body more clearly in remote sensing imagery, RGB imagery is false color composite with band 4, band 3 and band 2 in TM5 imagery, also make the NDVI imagery through band 3 and band 4 for next step.

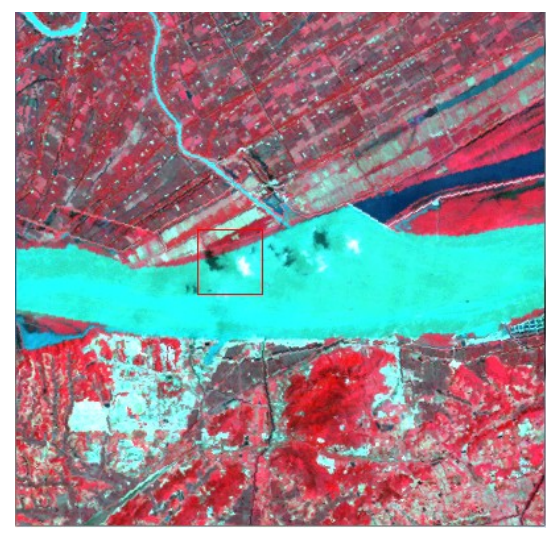

$\operatorname{RGB}(4,3,2)$

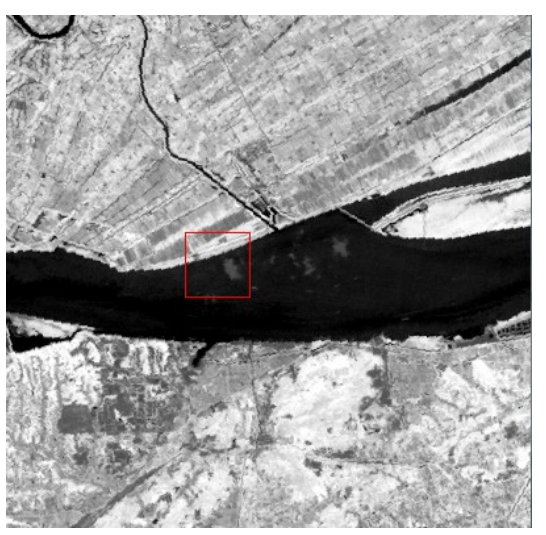

NDVI

Fig. 3. The contrast results between NEW and VNDWI 

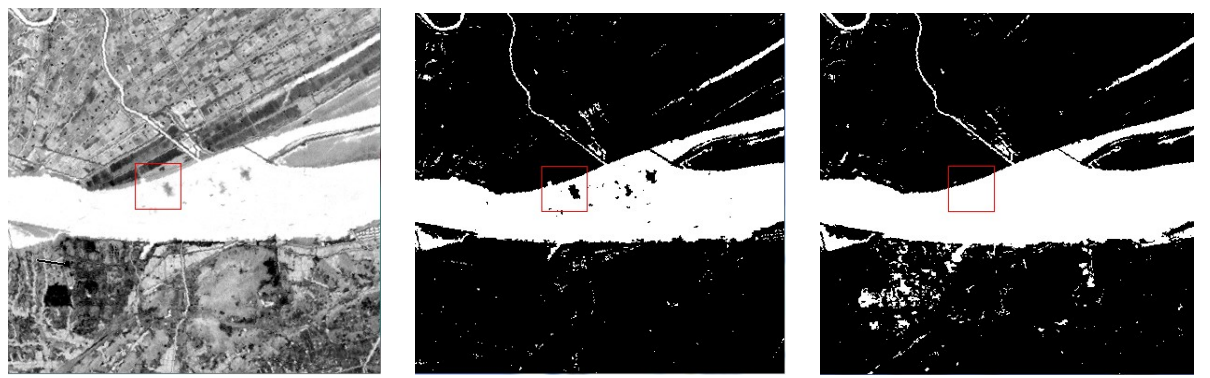

NEW

Binarization results of NEW Binarization results of VNDWI

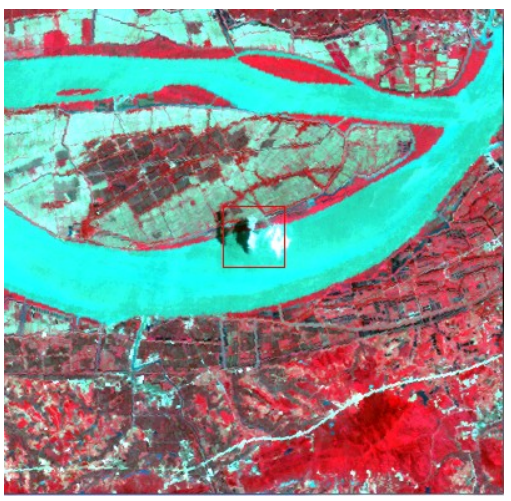

$\operatorname{RGB}(4,3,2)$

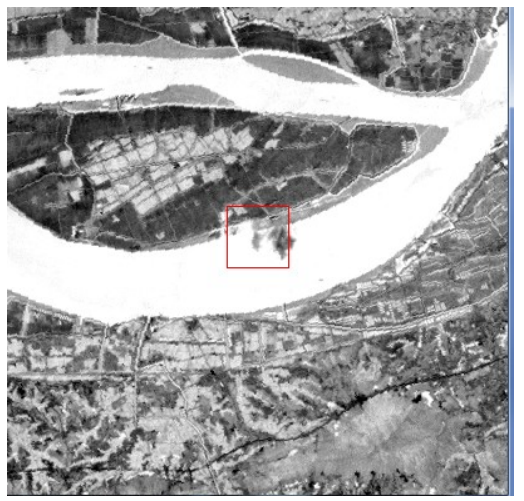

NDVI

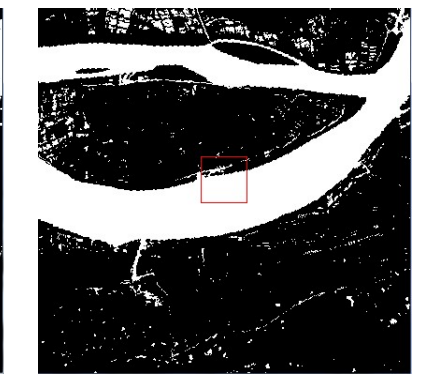

Binarization results of VNDWI

NEW

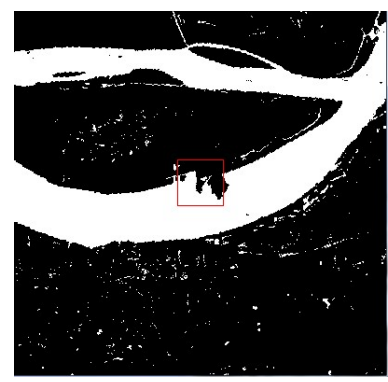

Binarization results of NEW

Fig. 3. (Continued)

After process from the NDVI imagery, we can see it has eliminate a little influence from the RGB imagery, then let k equals 100 in this particular study area, and from formula 1, we can get the binarization results of NEW and VNDWI from Figure3, which compares NEW and VNDWI imagery, it shows that VNDWI eliminates the influences of cloud and clouds' shadow from the binarization imagery, and buildings and subsequent shadow elimination is well preserved, make the extraction more precisely in the cloud cover area. 


\section{Conclusion}

Use Band 1 and Band 7 from Landsat TM5 imagery to build up the water index NEW, can be used for rapid, simple and accurate extraction a wide range of water body information, like the NDWI, MNDWI methods, can extract the vegetation water, also it can be better than NDWI and MNDWI more accurate extraction in the city area; But the NEW algorithm is not pointed to consider clouds influences in water extraction. However, this paper put forward a improved method called VNDWI algorithm, to some extent, it filter out the cloud influence from water body, and also keep its buildings and mountain shadows filtering characteristics, greatly improving the water body information extraction accuracy.

Acknowledgements. The research is funded by the "Eleventh Five-Year" national satellite application of high technologies in China. All authors would like to appreciate many experts from Office of the State Council Three Gorges Project Construction Committee, Beihang University and Chongqing Normal University.

\section{References}

1. Jensen, O.: Knowledge based classification of an urban area using texture and context information in Landsat TM imagery. Photogrammetric Engineering and Remote Sensing 56 (1990)

2. Mc Feeters, S.K.: The use of the Normalized Difference Water Index (NDWI) in the delineation of open water feature. International Journal of Sensing (1996)

3. Xu, H.-Q.: A Study on Information Extraction of Water Body with the Modified Normalized Difference Water Index. Journal of Remote Sensing (2005)

4. Yang, C.-J., Xu, M., et al.: Remote sensing information mechanism of water extraction method. Geography Research, 86-89 (1998)

5. Xiao, Y.-F., Zhu, L.: A study on information extraction of water body using band 1 and band7 of TM imagery. Surveying and Mapping (2010)

6. Zhao, S.-Y.: Principles and methods of Remote Sensing Applications. Science Press, Beijing (2003) 